\title{
Integrating substance abuse care with community diabetes care: implications for research and clinical practice
}

This article was published in the following Dove Press journal:

Substance Abuse and Rehabilitation

10 January 2012

Number of times this article has been viewed

\author{
Udi E Ghitza' \\ Li-Tzy Wu ${ }^{2}$ \\ Betty Tai ${ }^{\prime}$ \\ 'Center for the Clinical Trials \\ Network, National Institute on Drug \\ Abuse, National Institutes of Health, \\ United States Department of Health \\ and Human Services, Bethesda, MD, \\ ${ }^{2}$ Department of Psychiatry and \\ Behavioral Sciences, Duke University \\ Medical Center, Durham, NC, USA
}

Correspondence: Udi E Ghitza Center for the Clinical Trials Network, National Institute on Drug Abuse, National Institutes of Health, 600I Executive Boulevard, Bethesda, MD 20892, USA

Tel +I 30I 4436697

Fax + I 30I 4432317

Email ghitzau@nida.nih.gov
Abstract: Cigarette smoking and alcohol use are prevalent among individuals with diabetes in the US, but little is known about screening and treatment for substance use disorders in the diabetic population. This commentary discusses the scope and clinical implications of the public health problem of coexisting substance use and diabetes, including suggestions for future research. Diabetes is the seventh leading cause of death in the US, and is associated with many severe health complications like cardiovascular disease, stroke, kidney damage, and limb amputations. There are an estimated 24 million adults in the US with type 2 diabetes. Approximately $20 \%$ of adults aged 18 years or older with diabetes report current cigarette smoking. The prevalence of current alcohol use in the diabetic population is estimated to be around $50 \%-60 \%$ in epidemiological surveys and treatment-seeking populations. Cigarette smoking is associated with an increased risk of type 2 diabetes in a dose-dependent manner and is an independent modifiable risk factor for development of type 2 diabetes. Diabetic patients with an alcohol or other drug use disorder show a higher rate of adverse health outcomes. For example, these patients experience more frequent and severe health complications as well as an increased risk of hospitalization, and require longer hospital stays. They are also less likely to seek routine care for diabetes or adhere to diabetes treatment than those without an alcohol or other drug use disorder. The Affordable Care Act of 2010 and the Mental Health Parity Act and Addiction Equity Act of 2008 provide opportunities for facilitating integration of preventive services and evidence-based treatments for substance use disorders with diabetes care in community-based medical settings. These laws also offer emerging areas for research.

Keywords: addiction, illicit drug use, substance use disorder, substance abuse treatment, alcohol use, diabetes care, primary care, screening, brief intervention

\section{Introduction}

Substance use (ie, use of tobacco products, alcohol, illicit drugs, nonmedical use of prescription drugs) is relatively common among individuals presenting for primary care in general medical settings (including persons with type 2 diabetes) in the US, but substance use disorders have been grossly underdetected or undertreated with effective, evidence-based care. ${ }^{1-3}$ In 2010 , there were an estimated 20.5 million individuals, or $8.1 \%$ of the noninstitutionalized US population aged 12 years or older, who needed but did not receive substance abuse treatment services for problems related to alcohol or illicit drug use at a specialty facility in the past year. ${ }^{2}$ This estimate has not changed significantly between 2002 and 2010, ${ }^{2}$ indicating a pervasive pattern of unmet need for substance abuse care. This pattern of low rates of treatment use is consistent with treatment admission data, which show that the majority of persons with a substance use 
disorder delay seeking treatment for over a decade following onset of their primary substance abuse. ${ }^{3,4}$ Given that effective treatments for substance use disorder are available and that substance use and related problems are among the leading preventable causes of emergency department visits and mortality, ${ }^{5,6}$ undertreatment for substance use disorder is of major public health concern. Of note, substance abuse can affect almost every major organ, including the cardiovascular system and kidneys. ${ }^{7}$

Individuals with diabetes also have comparatively high rates of medical conditions, including cardiovascular disease and kidney damage. ${ }^{8}$ Persons with comorbid substance abuse and diabetes have a particularly high risk for developing and experiencing medical comorbidities and hospital readmissions. ${ }^{9,10}$ Specifically, diabetic patients with a coexisting substance use disorder have been found to have more adverse outcomes and poorer adherence to diabetes care than those without a substance use disorder. ${ }^{11,12}$ The available evidence also shows that alcohol abuse and cigarette smoking are associated with elevated odds of developing type 2 diabetes. ${ }^{13,14}$ The objective of this commentary is to discuss the clinical implications of the public health problem of coexisting substance use-related problems and diabetes, with an emphasis on the need for evidence-based, effective screening for substance use and treatment to improve the quality of care for persons with diabetes. Another aim is to stimulate future research in this area. Due to the paucity of research on illicit drug use in diabetic patients, we focus on cigarette smoking and alcohol use and their potential effects on diabetes care. In light of current health care reform, research is needed to inform integration of preventive services for substance use disorder and treatment of diabetes in primary care settings.

\section{Cigarette smoking and diabetes}

In 2010, approximately $20 \%$ of American adults aged 18 years or older with diabetes reported current cigarette smoking, and this prevalence has remained stable since $1994 .{ }^{15}$ Findings from a recent systematic literature review have shown that cigarette smoking is associated with an increased risk of diabetes in a dose-dependent manner and is an independent modifiable risk factor for development of diabetes. ${ }^{13}$ Furthermore, cigarette smoking heightens the risk for diabetic neuropathy, nephropathy, retinopathy, and peripheral vascular disease. ${ }^{16}$ Multiple studies have also found an association between cigarette smoking and smoking-induced hyperglycemia, hyperinsulinemia, elevated blood pressure, ${ }^{17}$ and impaired endothelial function ${ }^{18}$ (risk factors for type 2 diabetes). On the other hand, early smoking cessation has been shown to decrease the risk of development of type 2 diabetes to the level of nonsmokers. ${ }^{19}$ Smoking cessation also reduces the risk of coronary heart disease and mortality among these patients. ${ }^{20}$ Moreover, smoking cessation treatment has been found to be a cost-effective means to enhance treatment of type 2 diabetes among patients with coexisting type 2 diabetes and nicotine dependence (for example, $\leq \$ 25,000$ per life year gained or quality-adjusted life year). ${ }^{21,22}$ Therefore, the evidence suggests that there is a need for health care professionals to ask patients with type 2 diabetes about tobacco use and to offer effective smoking cessation counseling or treatment, as appropriate, given the considerable health risks and complications associated with cigarette smoking in these patients. Furthermore, screening for cigarette smoking and appropriate smoking cessation interventions are also critical in adolescents with type 2 diabetes, particularly given that many of them begin tobacco use after being diagnosed with diabetes. ${ }^{23,24}$ In summary, cigarette smoking increases the risk of developing adverse health effects such as cardiovascular problems, neuropathy, and kidney damage. There are notable research gaps, which need to be filled. These include development of costeffective and effective interventions to integrate preventive screening and smoking cessation treatment into communitybased diabetes care, in a manner that can address both cigarette smoking prevention and diabetes care.

\section{Alcohol use and diabetes}

Adults who have been diagnosed with diabetes are at increased risk of cardiovascular disease, and problematic alcohol use may exacerbate the problem. ${ }^{25}$ The prevalence of current alcohol use in the diabetic population has been estimated to be around $50 \%-60 \%$ in epidemiological surveys and treatment-seeking populations. ${ }^{1,26}$ Binge drinking of alcohol is especially associated with an increased risk of type 2 diabetes. ${ }^{14,27}$ In a systematic review, binge drinking of alcohol (defined as four or more drinks per day) was associated with a $43 \%$ increase in the risk of developing diabetes. ${ }^{14}$ Binge drinking of alcohol has also been found to precipitate diabetic ketoacidosis and to be an independent cause of peripheral neuropathy and retinopathy. Coexisting diabetes and heavy alcohol use can have synergistic effects for these medical complications. ${ }^{25}$ Moreover, alcohol consumption was inversely associated with adherence to diabetes self-care behaviors. ${ }^{26}$ Collectively, these findings underscore the importance of assessing and medically managing problematic alcohol use among individuals with diabetes using 
validated alcohol use assessments to identify alcohol use early. Such assessments are needed to incorporate alcohol-related interventions into diabetes care. However, there is very little research conducted in this area. The high prevalence of alcohol use in the diabetic population and its association with alcohol-related exacerbation of diabetes and medical complications indicate the need for research to identify effective preventive interventions for heavy or harmful alcohol use. Such interventions need to be integrated with diabetes care in order to reduce the risks of alcohol-related complications in patients with type 2 diabetes.

\section{Substance abuse and diabetes treatment outcomes}

The need to address alcohol use in the diabetes population is further supported by study findings showing that individuals with coexisting type 2 diabetes and a substance use disorder have poorer clinical outcomes and worse adherence to diabetes treatment than those without a substance use disorder. ${ }^{11,12}$ For example, a recent large retrospective study analyzed pooled Medicare and Medicaid claims data from Massachusetts in 2004 and 2005. It examined whether Medicare and/or Medicaid beneficiaries with type 2 diabetes and a coexisting substance use disorder had higher rates of type 2 diabetes-related complications and hospitalizations relative to type 2 diabetic patients without a coexisting substance use disorder. ${ }^{11}$ Medicaid and Medicare beneficiaries with an alcohol or other drug use disorder had elevated rates of adverse outcomes. Specifically, they were less likely than other groups to seek out routine treatment for diabetes care, had more frequent and severe health complications, an increased risk of hospitalization, and required longer hospital stays. ${ }^{11}$ Another study that also used the same dataset examined patient adherence with measures of quality of type 2 diabetes care among Medicare and/or Medicaid beneficiaries with type 2 diabetes, using an integrated data set of Medicare and Medicaid claims from Massachusetts in 2005. ${ }^{12}$ The presence of an alcohol or other drug use disorder was found to be associated with having lower odds of full adherence with measures of quality of care for type 2 diabetes compared with other groups without a substance use disorder. ${ }^{12}$ In conclusion, these findings suggest that not only do individuals with coexistent type 2 diabetes and a substance use disorder have substantially greater odds of adverse health outcomes compared with other groups, but they are also less likely to adhere to diabetes care. These factors may compromise the impact of treatment of type 2 diabetes to improve overall health outcomes in these individuals if the substance use disorder is left untreated.

\section{Impact of Affordable Care Act on treatment of substance abuse and diabetes}

Like diabetes, substance use disorder is considered a chronic disease, which should be monitored and managed clinically over a long period of time to reduce the risk of relapse and promote full recovery. ${ }^{28,29}$ The majority of individuals visit clinicians for general health care or medical conditions annually. Therefore, there are many opportunities in general medical settings for health care professionals to provide substance-using patients with screening, intervention, and referral to appropriate specialty treatment services to lessen the likelihood of substance abuse-related adverse events. ${ }^{30}$

Colocated or team-based integrated care for mental and medical health conditions has been shown to improve the coordination of care and patient outcomes for patients with multiple coexistent chronic illnesses. ${ }^{31,32}$ A team-based care approach is one in which nonphysicians (eg, medical assistants, social workers, nurses, psychologists, case managers) can be trained to partner with the physician to improve patient care by coordinating their interventions and tracking outcomes in a uniform, coordinated manner, as in a patient-centered medical home. ${ }^{33}$ Such a team-based care approach has been shown to increase screening and quit rates for nicotine dependence and for harmful alcohol use. ${ }^{33,34}$ In a patient-centered medical home and similar team-based care approach, there are several central features of processes which enhance treatment outcomes for patients with coexistent chronic conditions. ${ }^{33,34}$ These include: an emphasis on evidence-based patient-centered (rather than condition-centered) care that stresses timely coordination of care among a team of providers; use of health information technology to furnish point-of-care clinical information and clinical decision support to all members of the care team at different sites and over extended time frames, to track care optimally in a mutually compatible manner; attention to quality, safety, and clinical performance improvement processes using validated clinical quality measures (such as National Quality Forum-endorsed measures) as performance metrics to track and enforce accountability in delivering quality care; and managing care using evidence-based guidelines for chronic care management. More details about this approach are described later. Studies evaluating integrated care services using a teambased care approach for treating substance use disorder and associated medical conditions have shown that such care can be cost-effective and lead to lower total medical costs and 
improved health outcomes. ${ }^{35-41}$ Several models for coordinating substance use disorder and other medical care have been shown to be successfully implemented in a variety of patient populations and settings. ${ }^{35-41}$ They included adult Medicaid patients in hospital emergency department and community health clinic settings, adult patients in a private integrated health plan (Kaiser Permanente) receiving integrated medical and chemical dependency care, and adult patients entering treatment at the outpatient chemical dependency recovery program in Kaiser-Permanente Sacramento. ${ }^{35-37,39,41}$ Another study included adult welfare beneficiaries who were screened for a substance use disorder and, if screened positive, randomized to receive coordinated management or provided with usual care. ${ }^{38}$ A separate study included adult patients from 20 primary care clinical sites across the state of Wisconsin. ${ }^{40}$ For instance, in a randomized controlled trial, Parthasarathy et al demonstrated that in patients with substance use-related physical or psychiatric comorbidities, an integrated care model, in which primary care services were included in a drug abuse treatment program, was more effective than nonintegrated care in reducing hospitalization rates, utilization of emergency department visits, and inpatient care.$^{37}$ In primary care settings, substance abuse screening, brief intervention, and referral to treatment (SBIRT) for harmful alcohol use and other drug use disorders has been shown to save health costs. For example, a SBIRT program in Washington State in the US was estimated to save the Medicaid health insurance program approximately $\$ 366$ per member per month for all patients, which included patients who received a referral for treatment of a substance use disorder. ${ }^{35}$ Importantly, another large-scale study demonstrated that expanding alcohol and other drug abuse treatment in the US to include large numbers of Medicaid insurance beneficiaries achieved a substantial return on investment, with an impressive 2:1 ratio of 2 US dollars in medical and nursing facility costs saved per 1 US dollar invested in this expanded substance use disorder treatment. ${ }^{41}$ Of note, the Affordable Care Act (ACA) of 2010 emphasizes the expansion of preventive services and clinical management for behavioral health conditions. ${ }^{42}$ Examples of these services include substance use screening, health risk assessment, brief intervention, counseling services, and clinical treatment for substance use-related conditions (eg, services for tobacco smoking cessation, intervention for harmful alcohol use, and office-based treatment for substance use disorder). ${ }^{42}$ Tobacco smoking cessation services to be covered by ACA, in a manner comparable with other essential health benefits, include those that the US Preventive Health Services Task Force recommends. ${ }^{34,42}$ These are that clinicians are recommended to screen all adults and pregnant women for tobacco use and to provide tobacco cessation interventions for those who use tobacco products. ${ }^{34,42}$

The ACA also supports the integration of substance abuse care into general medical settings. ${ }^{42}$ In short, with full enactment of the ACA, along with support of insurance coverage for substance abuse care from the Mental Health Parity Act and Addiction Equity Act of 2008 (http://www.samhsa.gov/ healthreform/parity/), preventive services for substance use disorder are considered an essential part of routine care. Moreover, treatment for substance use disorder in general medical settings is expected to be managed like other chronic medical illnesses. ${ }^{42}$ The ACA provisions have been estimated to expand health insurance coverage to about 32 million Americans who are currently uninsured, including persons with substance use disorders and/or other chronic medical conditions (like type 2 diabetes). ${ }^{42}$ Under the ACA and the Mental Health Parity Act, substance abuse services and treatment are expected to become more patient-centered in the coming years, resulting from an expanded role for Medicaid as a payer for millions of individuals suffering from substance use disorders and/or other coexisting chronic conditions in a recovery-oriented medical home care model. ${ }^{42}$ However, the success of these promising health care reform goals will be influenced by whether individuals presenting for health care in general medical settings are screened for substance use and related problems with validated or standardized screening questions or tools. ${ }^{43}$ Such screening would preferably be conducted using validated combined instruments that, in a bundled manner, assess illicit drug use, nonmedical use of prescription drugs, harmful alcohol use, and tobacco smoking in tandem. ${ }^{43}$ These goals will also be enhanced if such assessments are embedded with clinical decision support for brief onsite intervention and linkage with referrals to specialty treatment programs into the existing electronic health record system to facilitate workflow and integration of substance abuse treatment with primary care. ${ }^{43}$

One key factor, which can help to achieve adoption and implementation of SBIRT by primary care providers, is incorporation of the standardized and validated screening tools for substance use disorder as common data elements with point-of-care clinical decision support tools into the electronic health records of general medical settings. ${ }^{44}$ Common data elements for substance use disorder can be utilized as electronic tools to provide a metadata-based terminology to enable uniform collection and exchange of health information data relevant to substance use disorder in electronic health records across many platforms in a standardized, semantically rich format. ${ }^{44}$ 
Standardized and validated electronic screening tools are needed in the electronic health records of primary care settings to facilitate identification of patients with substance use problems and tracking of patients' progress and health outcomes over time. There is an urgent need to develop and field-test such tools. ${ }^{44}$ For example, the National Institute on Drug Abuse Clinical Trials Network has collaborated with and sought buy-in of federal, state, and community stakeholders in community health centers and other general medical settings on development of brief SBIRT screening tools, which could be incorporated into electronic health records. ${ }^{44}$ These SBIRT tools have been developed with an accompanying expert-defined, consensus-based clinical decision support algorithm to guide the disposition of patients. ${ }^{44}$ This involves a bottom-up, consensus-building approach with an iterative input from many stakeholders participating in workshops organized by the Clinical Trials Network on this initiative over an 18-month period. Details regarding this process are described elsewhere. ${ }^{44}$

Future research is needed to test these standardized electronic screening and assessment tools to evaluate the efficacy and cost-effectiveness of SBIRT for substance use disorder in improving health outcomes and reducing health care costs for individuals with coexisting substance use disorder and diabetes. As reviewed in the studies above, there is a pervasive pattern of unmet need for treatment of substance abuse disorders. There are also dire consequences from untreated substance use disorders on the medical complications of diabetes, medical adherence with diabetes care, and other health outcomes in these patients. Therefore, it is necessary that health care providers in general medical settings be provided with appropriate training and resources as well as reimbursement incentives, to support and guide evidencebased SBIRT for substance use disorders. These measures should be instituted as a means to improve the detection, medical management, and referral to the specialty services needed in diabetic individuals with a substance use disorder. This would furnish these providers with tools and resources which play a critical role in coordinating and implementing screening for substance use disorder and medical management in patients with coexisting substance use disorder and other medical comorbidities, such as diabetes..$^{30,45-47}$

It is widely anticipated that promoting integration of general medical care with behavioral health care services will continue to be a key part of ACA health care reform in the coming years. ${ }^{30}$ Diabetes patients with a coexisting substance use disorder have substantially higher odds of adverse medical outcomes and show poorer adherence with diabetes treatment than those without an substance use disorder. As reviewed above, to improve the clinical course and treatment response in patients with diabetes, early screening is needed to enhance detection and timely treatment for substance use disorder in general medical settings. In this regard, community-based translational or implementation research may be utilized to test the effects of integrating SBIRT for substance use disorder with diabetes care. Implementation science research on how to implement strategies for integrating SBIRT for alcohol and other drug use problems into diabetes care models in a cost-effective manner is needed. One direction may be to utilize a chronic care management model, for example, the framework specified in the standards for patient-centered medical homes devised by the National Committee for Quality Assurance as well as established performance measures for delivering quality care from the Healthcare Effectiveness Data and Information Set. ${ }^{48,49}$ These standards provide the criteria for guiding and measuring quality of health care and coordination, involving team management of chronic diseases across primary and behavioral health care providers, which is particularly relevant for patients with multiple chronic coexisting conditions. These standards are defined across six categories: enhancing care access and continuity of care (both of which are important to patients with coexisting disorders such as diabetes and substance use disorder); collecting population level data for identifying and managing patient populations (also pertinent to this patient population); planning and managing care using evidence-based guidelines for preventive, acute, and chronic care (including medications) management (including identifying patients with high-risk conditions, such as diabetes or substance abuse problems); providing self-care support and community resources (including assessing and providing or arranging for substance abuse treatment in patients with coexisting conditions, including comorbid substance use disorder and diabetes); utilizing health information technology, including electronic health records, to track, follow up on, and coordinate medical tests, referrals, and transitions of care (also essential to this patient population); evaluating performance of these, using measures for continuous quality improvement (pertinent to patients with coexisting diabetes and substance use disorder). ${ }^{48}$

In summation, this standards framework based on patient-centered medical homes describes how health information technology, health information exchange, and disease registries could be leveraged to ensure that patients with chronic comorbid conditions (such as diabetes and substance use disorder) receive appropriate coordinated 
care for their coexisting medical needs in a timely manner, based on the latest accreditation standards and performance measures. ${ }^{48}$ Public reporting of related performance measures relevant to assessing continuity and coordination of care as well as care transitions for patients with chronic diseases (for instance, substance use disorder and diabetes) will likely drive health service reimbursement by the Centers for Medicare and Medicaid Services in the coming years under the ACA (for examples, per ACA sections 10331 and 10332 on public reporting of performance information). ${ }^{50}$ In addition, Section 10305 of the ACA requires that public reporting of performance (quality measures) information to determine Centers for Medicare and Medicaid Services reimbursement (some of which involves quality measures pertinent to the patient-centered medical home model) must be aligned with the expansion, interoperability efforts, and standard setting of health information technology systems, including electronic health records. ${ }^{50}$ This section of the ACA directs the Secretary for the Department of Health and Human Services to ensure that data collection, data aggregation, and analysis systems for public reporting of performance (quality measures) information involve an "increasingly broad range of patient populations, providers, and geographic areas over time" (which includes those patient populations with diabetes and substance use disorder). ${ }^{50}$

The recommendations for priority health services research areas mentioned above align well with ongoing federal initiatives on integrated care and patient-centered medical homes, such as the "Medicaid State Option to Provide Health Homes for Enrollees with Chronic Conditions" from the Centers for Medicare and Medicaid Services and the "Primary Care and Behavioral Health Care Integration Program" from the Substance Abuse and Mental Health Services Administration. Moreover, Section 2703 of the ACA incentivizes state Medicaid programs to provide integrated care in patient-centered medical homes for patients with chronic coexisting conditions, including substance use disorder and diabetes. ${ }^{50}$ It also incentivizes these Medicaid programs to have a health home option under which states can reimburse a patient-designated health home provider who meaningfully utilizes health information technologies to monitor and coordinate service providers involved in integrated treatment delivery. ${ }^{50}$ Furthermore, such standards for quality integrated care consistent with patient-centered medical homes have been endorsed by major medical societies, funders, and health systems, and applied across a wide variety of clinical settings, showing positive outcomes. ${ }^{51-53}$ ACA section 1302 also stipulates that any health insurance plan in the US should cover mental health or substance use disorder services as essential health benefits, comparable with other essential health benefits. ${ }^{50}$ SBIRT is well suited to implementation within a team-based patient-centered medical home approach. For instance, social workers, nurses, or medical assistants can conduct rapid, simple, and standard screenings for harmful substance use, and implement brief motivational interventions to reduce identified use. ${ }^{46}$

\section{Conclusion}

This commentary illuminates a public health need for future research in six emerging areas (proposed below) for the development of cost-effective interventions to integrate preventive screening and substance use disorder treatment into community diabetes care, in a manner which considers both prevention of substance use disorder and management of diabetes. First, future research is needed to assess the outcomes and cost-effectiveness of SBIRT and integrated treatment for substance use disorder and primary care services in the diabetic population within the context of an ACA-consistent framework for integration of care, such as the patient-centered medical home or other chronic care disease management model. Second, measures for program durability and performance (eg, clinical quality measures) of models for SBIRT delivery should be built into the design of future research and delivery of care. Third, collaboration between federal agencies and community-based treatment providers to promote research on treatment of substance use disorder would be a useful means of helping to develop and test implementation strategies on how community health clinics and other general medical settings could effectively address the integration of community-based substance use disorder and diabetes care. Fourth, health services research is also needed in the context of current health care reform to examine how such an integrated or medical home model could be sustainable over time at an organizational/systems level. Fifth, future comparative effectiveness research is needed to compare the outcomes and cost-effectiveness of different care management team composition strategies for managing type 2 diabetes optimally in tandem with the spectrum of substance use seen within primary care settings. Sixth, future research is needed on how to integrate treatment for substance use disorder into chronic care management models for treating type 2 diabetes and other chronic diseases in a cost-effective and patient-centered (as opposed to a symptom-specific or condition-specific) manner longitudinally over time. This health services research necessitates examining how to implement clinical informatics systems efficiently in support of 
team-based patient-centered treatment suitable for chronic management of patients with these coexisting conditions. Such integrative care, when shown to be effective and sustainable in general medical settings, would greatly benefit the overall health and course of care in individuals with type 2 diabetes who have substance use problems.

\section{Acknowledgments}

UEG and BT are employees of the Center for the Clinical Trials Network, National Institute on Drug Abuse, National Institutes of Health, which is the funding agency for the National Drug Abuse Treatment Clinical Trials Network. L-TW is affiliated with Duke University Medical Center. The opinions in this manuscript are those of the authors and do not represent the official position of the US government or Duke University.

\section{Disclosure}

The authors report no conflicts of interest in this work.

\section{References}

1. Lethbridge-Cejku M, Schiller JS, Bernadel L. Summary Health Statistics for US Adults: National Health Interview Survey, 2002. Report number 10. Hyattsville, MD: National Center for Health Statistics; 2004. Available from: http://www.cdc.gov/nchs/data/series/sr_10/sr10_225. pdf. Accessed December 18, 2012.

2. Substance Abuse and Mental Health Services Administration. Results from the 2010 National Survey on Drug Use and Health: summary of national findings. NSDUH Series H-41, HHS Publication No (SMA) 11-4658. Rockville, MD: Substance Abuse and Mental Health Services Administration; 2011. Available from: http://www.samhsa.gov/ data/nsduh/2k10nsduh/2k10results.htm. Accessed December 18, 2012.

3. Substance Abuse and Mental Health Services Administration, Center for Behavioral Health Statistics and Quality. The TEDS Report: length of time from first use to adult treatment admission. Rockville, MD: Substance Abuse and Mental Health Services Administration; 2011. Available from: http://www.samhsa.gov/data/2k11/WEB_TEDS_026/ Lngth_of_Time_FirstUse_to_AdultAdmsn.pdf. Accessed December 8, 2012.

4. Kessler RC, Olfson M, Berglund PA. Patterns and predictors of treatment contact after first onset of psychiatric disorders. Am J Psychiatry. 1998; 155:62-69.

5. Centers for Disease Control and Prevention. Emergency department visits involving nonmedical use of selected prescription drugs - United States, 2004-2008. MMWR Morb Mortal Wkly Rep. 2010;59:705-709.

6. Centers for Disease Control and Prevention. Unintentional drug poisoning in the United States. Available at: http://www.cdc.gov/ HomeandRecreationalSafety/pdf/poison-issue-brief.pdf. Accessed August 26, 2012.

7. Brick J. Handbook of the Medical Consequences of Alcohol and Drug Abuse. New York, NY: The Haworth Press; 2004.

8. Centers for Disease Control and Prevention. National Diabetes Fact Sheet: national estimates and general information on diabetes and prediabetes in the United States, 2011. Atlanta, GA: US Department of Health and Human Services, Centers for Disease Control and Prevention; 2011. Available from: http://www.cdc.gov/diabetes/pubs/factsheet11.htm. Accessed December 18, 2012.

9. Wadland WC, Ferenchick GS. Medical comorbidity in addictive disorders. Psychiatr Clin NAm. 2004;27:675-687.
10. Coffey RM, Misra A, Barrett M, Andrews RM, Mutter R, Moy E. Congestive heart failure: who is likely to be readmitted? Med Care Res Rev. 2012;69:602-616.

11. Leung GY, Zhang J, Lin W, Clark RE. Behavioral disorders and diabetes-related outcomes among Massachusetts Medicare and Medicaid beneficiaries. Psychiatr Serv. 2011;62:659-665.

12. Leung GY, Zhang J, Lin W, Clark RE. Behavioral health disorders and adherence to measures of diabetes care quality. Am J Manag Care. 2011;17:144-150.

13. Willi C, Bodenmann P, Ghali WA, Faris PD, Cornuz J. Active smoking and the risk of type 2 diabetes: a systematic review and meta-analysis. JAMA. 2007;298:2654-2664.

14. Howard AA, Arnsten JH, Gourevitch MN. Effect of alcohol consumption on diabetes mellitus: a systemic review. Ann Intern Med. 2004;140:211-219.

15. Centers for Disease Control and Prevention. Data and trends - risk factors for complications - age-adjusted percentage of adults aged 18 years or older with diagnosed diabetes who currently smoke, United States, 1994-2010. Available at: http://www.cdc.gov/diabetes/statistics/comp/ fig7_smoking.htm. Accessed November 5, 2012.

16. Eliasson B. Cigarette smoking and diabetes. Prog Cardiovasc Dis. 2003;45:405-413.

17. Frati AC, Iniestra F, Ariza CR. Acute effect of cigarette smoking on glucose tolerance and other cardiovascular risk factors. Diabetes Care. 1996;19:112-118.

18. Celermajer DS, Sorensen KE, Georgakopoulos D, et al. Cigarette smoking is associated with dose-related and potentially reversible impairment of endothelium-dependent dilation in health young adults. Circulation. 1993;88:2149-2155.

19. Hur NW, Kim HC, Nam CM, Jee SH, Lee HC, Suh I. Smoking cessation and risk of type 2 diabetes mellitus: Korea Medical Insurance Corporation Study. Eur J Cardiovasc Prev Rehabil. 2007;14:244-249.

20. Al-Delaimy WK, Manson JE, Solomon CG, et al. Smoking and risk of coronary heart disease among women with type 2 diabetes mellitus. Arch Intern Med. 2002;162:273-279.

21. Earnshaw SR, Richter A, Sorensen SW, et al. Optimal allocation of resources across four interventions for type 2 diabetes. Med Decis Making. 2002;22 Suppl 5:S80-S91.

22. Li R, Zhang P, Barker LE, Chowdhury FM, Zhang X. Cost-effectiveness of interventions to prevent and control diabetes mellitus: a systematic review. Diabetes Care. 2010;33:1872-1894.

23. Reynolds K, Liese AD, Anderson AM, et al. Prevalence of tobacco use and association between cardiometabolic risk factors and cigarette smoking in youth with type 1 and type 2 diabetes mellitus. J Pediatr. 2011;158:594-601.

24. Shaw NJ, McClure RJ, Kerr S, Lawton K, Smith CS. Smoking in diabetic teenagers. Diabet Med. 1993;10:275-277.

25. Emanuele NV, Swade TF, Emanuele MA. Consequences of alcohol use in diabetics. Alcohol Health Res World. 1998;22:211-219.

26. Ahmed AT, Karter AJ, Liu J. Alcohol consumption is inversely associated with adherence to diabetes self-care behaviors. Diabet Med. 2006;23:795-802.

27. Seike N, Noda M, Kadowaki T. Alcohol consumption and risk of type 2 diabetes mellitus in Japanese: a systematic review. Asia Pac J Clin Nutr. 2008;17:545-551.

28. American Society of Addiction Medicine. Public policy statement: definition of addiction. http://www.asam.org/docs/publicy-policystatements/1definition_of_addiction_long_4-11.pdf?sfvrsn=2\#search ="definitionofaddictionlong". Accessed August 4, 2012.

29. Volkow N, Li TK. The neuroscience of addiction. Nat Neurosci. 2005;8: 1429-1430.

30. Pilowsky DJ, Wu LT. Screening for alcohol and drug use disorders among adults in primary care: a review. Subst Abuse Rehabil. 2012;3: 25-34.

31. Druss BG, Rohrbaugh RM, Levinson CM, Rosenheck RA. Integrated medical care for patients with serious psychiatric illness: a randomized trial. Arch Gen Psychiatry. 2001;58:861-868. 
32. Druss BG, von Esenwein SA. Improving general medical care for persons with mental and addictive disorders: systematic review. Gen Hosp Psychiatry. 2006;28:145-153.

33. Sullivan LE, Tetrault JM, Braithwaite RS, Turner BJ, Fiellin DA. A meta-analysis of the efficacy of nonphysician brief interventions for unhealthy alcohol use: implications for the patient-centered medical home. Am J Addict. 2011;20:343-356.

34. Clinical Practice Guideline Treating Tobacco Use and Dependence 2008 Update Panel, Liaisons, and Staff. A clinical practice guideline for treating tobacco use and dependence: 2008 update. A US Public Health Service report. Am J Prev Med. 2008;35:158-176.

35. Estee S, Wickizer T, He L, Shah MF, Mancuso D. Evaluation of the Washington state screening, brief intervention, and referral to treatment project: cost outcomes for Medicaid patients screened in hospital emergency departments. Med Care. 2010;48:18-24.

36. Mertens JR, Risher AJ, Satre DD, Weisner C. The role of medical conditions and primary care services in 5-year substance use outcomes among chemical dependency treatment patients. Drug Alcohol Depend. 2008;98:45-53.

37. Parthasarathy S, Mertens J, Moore C, Weisner C. Utilization and cost impact of integrating substance abuse treatment and primary care. Med Care. 2003;41:357-367.

38. Morgenstern J, Hogue A, Dauber S, Dasaro C, McKay JR. A practical clinical trial of coordinated care management to treat substance use disorders among public assistance beneficiaries. J Consult Clin Psychol. 2009; 77:257-269.

39. Parthasarathy S, Chi FW, Mertens JR, Weisner C. The role of continuing care in 9-year cost trajectories of patients with intakes into an outpatient alcohol and drug treatment program. Med Care. 2012;50:540-546.

40. Quanbeck A, Lang K, Enami K, Brown RL. A cost-benefit analysis of Wisconsin's screening, brief intervention, and referral to treatment program: adding the employer's perspective. WMJ. 2010;109:9-14.

41. Mancuso D, Felver BEM. Bending the health care cost curve by expanding alcohol/drug treatment. Olympia, WA: Research and Data Analysis Division, Department of Social and Health Services, Washington State Department of Social and Health Services; 2010.

42. Buck JA. The looming expansion and transformation of public substance abuse treatment under the Affordable Care Act. Health Aff (Millwood). 2011;30:1402-1410.
43. Tai B, Wu LT, Clark HW. Electronic health records: essential tools in integrating substance abuse treatment with primary care. Subst Abuse Rehabil. 2012;3:1-8.

44. Ghitza UE, Gore-Langton RE, Lindblad R, Shide D, Subramaniam G, Tai B. Common data elements for substance use disorders in electronic health records: the NIDA Clinical Trials Network experience. Addiction. May 8, 2012. [Epub ahead of print.]

45. US Preventive Services Task Force. Screening and behavioral counseling interventions in primary care to reduce alcohol misuse: recommendation statement. Ann Intern Med. 2004;140:554-556.

46. Madras BK, Compton WM, Avula D, Stegbauer T, Stein JB, Clark HW. Screening, brief interventions, referral to treatment (SBIRT) for illicit drug and alcohol use at multiple healthcare sites: comparison at intake and 6 months later. Drug Alcohol Depend. 2009;99: 280-295.

47. Levy SJ, Kokotailo PK; Committee on Substance Abuse. Substance use screening, brief intervention, and referral for treatment for pediatricians. Pediatrics. 2011;128:e1330-e1340.

48. National Committee for Quality Assurance. NCQA's Patient-Centered Medical Home (PCMH) 2011. Available from: http://www.ncqa.org/ tabid/631/Default.aspx. 2011. Accessed November 5, 2012.

49. National Committee for Quality Assurance. HEDIS and Quality Measurement. Available from: http:/www.ncqa.org/tabid/59/default. aspx. 2011. Accessed November 5, 2012.

50. Healthcare.gov. Read the Law|Healthcare.gov. The Affordable Care Act, Section by Section. Available from: http://www.healthcare.gov/ law/full/. 2012. Accessed November 5, 2012.

51. Rittenhouse DR, Casalino LP, Gillies RR, Shortell SM, Lau B. Measuring the medical home infrastructure in large medical groups. Health Aff (Millwood). 2008;27:1246-1258.

52. Kucukarslan SN, Hagan AM, Shimp LA, Gaither CA, Lewis NJ. Integrating medication therapy management in the primary care medical home: a review of randomized controlled trials. Am J Health Syst Pharm. 2011;68:335-345.

53. Wright MA, Knopf AS. Mobilizing a medical home to improve HIV care for the homeless in Washington, DC. Am J Public Health. 2009;99:973-975.
Substance Abuse and Rehabilitation

\section{Publish your work in this journal}

Substance Abuse and Rehabilitation is an international, peer-reviewed, open access journal publishing original research, case reports, editorials, reviews and commentaries on all areas of addiction and substance abuse and options for treatment and rehabilitation. The manuscript management system is completely online and includes a very quick and fair

\section{Dovepress}

peer-review system. Visit http://www.dovepress.com/testimonials.php to read real quotes from published authors. 